University of Nebraska - Lincoln

DigitalCommons@University of Nebraska - Lincoln

Fall 11-20-2008

\title{
Temperature dependence of the training effect in exchange coupled ferromagnetic bilayers
}

\author{
Christian Binek \\ University of Nebraska, Lincoln, cbinek@unl.edu \\ Srinivas Polisetty \\ University of Nebraska-Lincoln, polisetty.srinivas@gmail.com \\ Sarbeswar Sahoo \\ University of Nebraska-Lincoln, sarbeswar@gmail.com
}

Follow this and additional works at: https://digitalcommons.unl.edu/physicsbinek

Part of the Physics Commons

Binek, Christian; Polisetty, Srinivas; and Sahoo, Sarbeswar, "Temperature dependence of the training effect in exchange coupled ferromagnetic bilayers" (2008). Christian Binek Publications. 62.

https://digitalcommons.unl.edu/physicsbinek/62

This Article is brought to you for free and open access by the Research Papers in Physics and Astronomy at DigitalCommons@University of Nebraska - Lincoln. It has been accepted for inclusion in Christian Binek Publications by an authorized administrator of DigitalCommons@University of Nebraska - Lincoln. 


\title{
Temperature dependence of the training effect in exchange coupled ferromagnetic bilayers
}

\author{
S. Polisetty, ${ }^{1}$ S. Sahoo, ${ }^{1}$ A. Berger, ${ }^{2}$ and Ch. Binek ${ }^{1, *}$ \\ ${ }^{1}$ Department of Physics and Astronomy and the Nebraska Center for Materials and Nanoscience, University of Nebraska-Lincoln, \\ Lincoln, Nebraska 68588-0111, USA \\ ${ }^{2}$ CIC nanoGUNE Consolider, E-20009 Donostia-San Sebastian, Spain
}

(Received 11 July 2008; revised manuscript received 15 September 2008; published 20 November 2008)

\begin{abstract}
The temperature dependence of the training effect is studied in an exchange coupled thin-film bilayer composed of a hard ferromagnetic pinning $(\mathrm{CoPtCrB})$ layer in proximity of a soft ferromagnetic pinned $(\mathrm{CoCr})$ layer. Interlayer exchange shifts the hysteresis loops of the soft layer along the magnetic-field axis. This shift is quantified by the bias field in far reaching analogy to the exchange bias field of conventional antiferromagnetic/ferromagnetic heterostructures. A ferromagnetic domain state induced in the hard layer experiences aging very similar to the training behavior of the antiferromagnetic domain state in conventional exchange bias systems. Training originates from changes in the spin structure of the pinning layer with consecutive magnetization reversals of the pinned layer. Here we perform a detailed investigation of the temperature dependence of the bias field and its training effect. Consecutively cycled hysteresis loops of the soft layer are measured at various temperatures. We also derive a theoretical description of the temperature dependence of the training effect which is in agreement with the experimental data.
\end{abstract}

DOI: 10.1103/PhysRevB.78.184426

PACS number(s): 75.60.-d, 75.70.Cn

\section{INTRODUCTION}

Exchange bias (EB) is a coupling phenomenon which can be observed when an antiferromagnet and an adjacent ferromagnet share a common interface. Exchange coupling at the interface of antiferromagnetic (AF)/ferromagnetic (FM) thin films gives rise to a unidirectional anisotropy. Among the variety of effects related to the EB phenomenon the shift of the magnetic hysteresis loop along the magnetic field is the most prominent. This loop shift is quantified by the exchange bias field $\mu_{0} H_{\mathrm{EB}}$.

The EB phenomenon was originally discovered more than 50 years ago by Meiklejohn and Bean. ${ }^{1,2}$ Since then EB has been observed in a vast variety of systems including $\mathrm{AF} / \mathrm{FM}$ and FM/ferrimagnetic thin-film heterostructures, AF/FM core shell nanoparticles, FM precipitates in AF and spin glass matrices, and spin valves; but details of its origin still remains elusive to date. ${ }^{3-7}$

Similar to exchange-spring magnets, ${ }^{8-11} \mathrm{AF}$ coupled bilayers of soft and hard FM films show exchange-induced coupling phenomena analogous to conventional EB heterolayers. ${ }^{412-14}$ The FM hard layer (HL) pins the magnetically soft layer (SL) and shifts its hysteresis loops along the magnetic-field axis. The shift is quantified by the bias field $\mu_{0} H_{B}$. In the case of AF (FM) coupling, $\mu_{0} H_{B}$ is positive (negative) when the HL magnetization is set in a positive magnetization state and vice versa when the HL magnetization is negative. Antiferromagnetically coupled HL/SL bilayers are not only important in magnetic recording technology but can also be used as model systems to study EB and its related effects. ${ }^{15-17} \mathrm{HL} / \mathrm{SL}$ systems have several advantages over conventional AF/FM systems. For example, a FM pinning layer provides unique experimental access to the change in its magnetization state. In addition, the dependence of the bias field on the pinning layer magnetization can be directly measured by simple magnetometry. ${ }^{15,16}$ Moreover, AF materials are naturally inert to applied magnetic fields which limit the control of the AF domain state. Hence, isothermal tuning of the EB field and its training is very much limited to rare AF/FM systems. ${ }^{18,19}$ The situation is different when the pinning layer couples strongly to an applied magnetic field as it does in HL/SL heterostructures.

Training effect (TE) is one of several commonly observed features associated with EB and biasing in HL/SL systems. It is defined as alteration of the EB/bias field upon cycling the system through consecutive hysteresis loops and is quantified by $\mu_{0} H_{\mathrm{EB} / B}$ vs $n$, where $n$ is the number of cycled loops. ${ }^{20-27}$ Training can be observed when the spin structure of the pinning layer is initially out of equilibrium and approaches the equilibrium spin configuration triggered via subsequent reversals of the pinned magnetization. ${ }^{17}$

Many investigations have been done on the EB TE which focus for instance on the influence of temperature, AF and FM film thicknesses, ${ }^{26,28}$ and dilution of the AF. ${ }^{13,29}$ The temperature dependence of the TE in conventional AF/FM systems is rather complex. ${ }^{30}$ Recent attempts to measure the correlation between aging of the interface magnetization in an AF pinning layer and the training of the EB field in AF/FM heterostructures faced serious difficulties because of the smallness of the excess magnetic moment in the AF pinning layer that gives rise to conventional EB. ${ }^{31,32}$ Theoretically the description of the $T$ dependence of the TE in $\mathrm{AF} / \mathrm{FM}$ systems is challenging due to the nontrivial relation between the AF order parameter and the magnetization. ${ }^{33}$ It is the AF interface magnetization which ultimately gives rise to the EB effect and its training behavior. Also, in these systems, proportionality between the moment at the interface and the AF bulk magnetic moment is a faintly motivated assumption. The latter is far more realistic in the case of a very thin FM pinning layer with a homogeneous spin structure along the normal of the film as demonstrated by the linearity of the effect. Recently it has been observed that small deviations from linearity can appear. ${ }^{34}$

In all FM coupled systems training is initialized by partial demagnetization of the HL. Interestingly, and as an experi- 


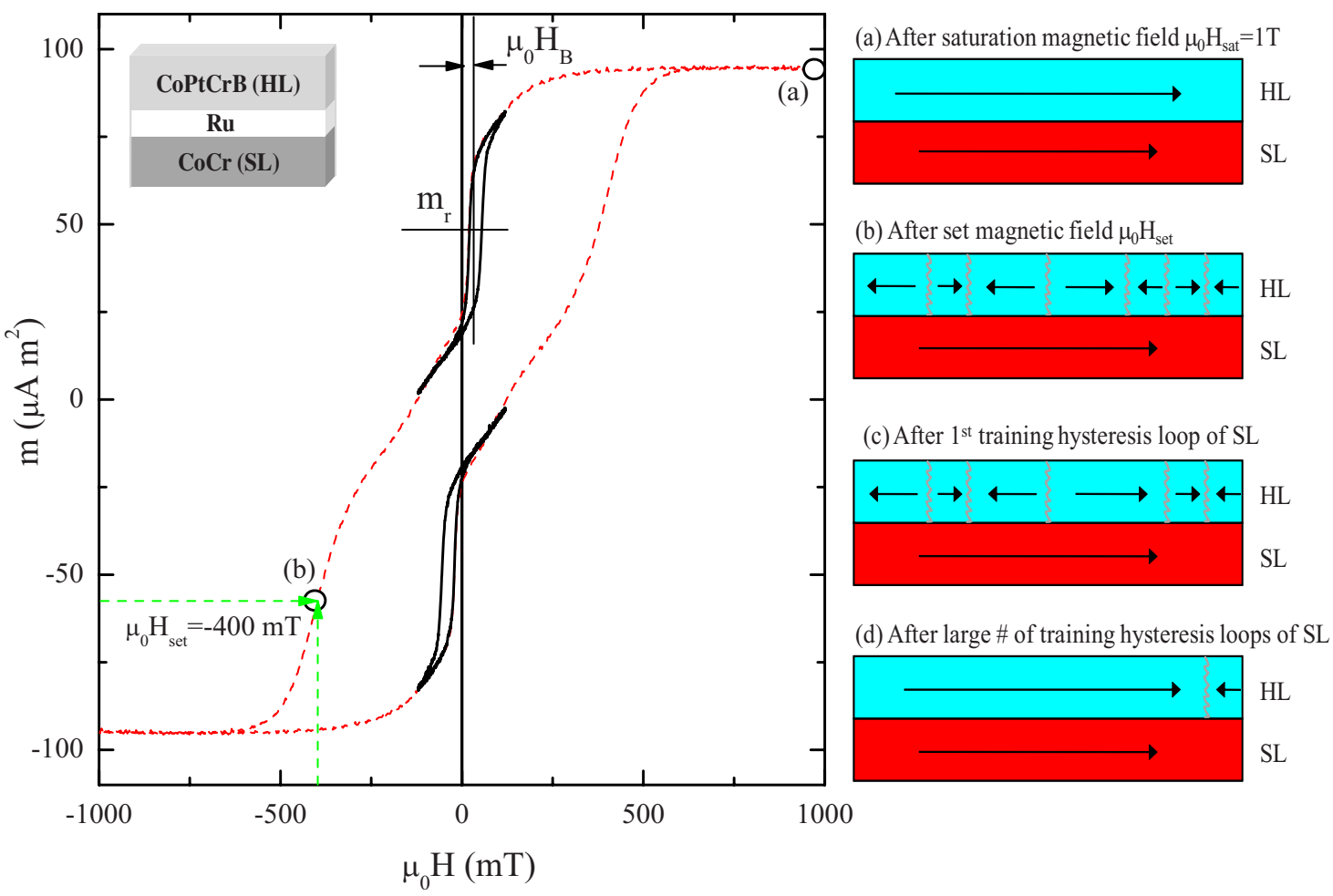

FIG. 1. (Color online) Overall magnetic hysteresis loop $m$ vs $\mu_{0} H$ (dotted red line). Solid black lines are typical minor (SL) loops after applying a set field of $H_{\text {set }}=-/+400 \mathrm{mT}$. The horizontal line visualizes $m_{r}$ for the upper SL loop, the vertical line indicate the shift of the SL loop along the field axis relative to $\mu_{0} H=0$. The inset is the schematic of the sample. The right frame sketches the magnetic domain state of HL/SL heterostructure at different stages during the training cycle.

mental big advantage, the HL magnetization can be isothermally tuned by a specific magnetic-field protocol. ${ }^{16}$ It is given by initial saturation and subsequent demagnetization of the HL unlike the tedious field-cooling procedure in AF/FM systems. Moreover, $T$ dependence of the TE is also expected in HL/SL systems due to the temperature dependence of the HL domain state and its thermally assisted approach toward equilibrium on SL cycling. Thus, coupled HL/SL heterostructures are intriguing systems to investigate various training related effects.

In this article, we report a systematic study of the $T$ dependence of bias field TE in all FM bilayers, in which a pinned SL is antiferromagnetically exchange coupled via a $\mathrm{Ru}$ intermediate layer with a pinning HL. We present a theory of the $T$ dependence of TE which shows excellent agreement with our experimental data. The work presented in this paper is structured as follows. In Sec. II we describe details of the sample, the experimental protocols, and the results of the measurements. In Sec. III we develop the theory, apply it to our experimental results, and bring it into context of our previous work including the training effect of AF/FM exchange bias systems. ${ }^{17,24,26,30,33}$ Finally we conclude in Sec. IV with an intuitive interpretation of our results.

\section{EXPERIMENTAL DETAILS AND RESULTS}

The SL of the sample under investigation is a $\mathrm{CoCr}$ film of $3 \mathrm{~nm}$ thickness. It is exchange coupled with a magneti- cally hard CoPtCrB pinning layer of $15 \mathrm{~nm}$ thickness via a $\mathrm{Ru}$ interlayer of thickness of $0.7 \mathrm{~nm} .{ }^{17}$ Details of the sample fabrication can be found elsewhere. ${ }^{15,16}$ In the left frame of Fig. 1 the dotted line shows the overall magnetic hysteresis loops $m$ vs $\mu_{0} H$, where $m$ is the magnetic moment and $H$ is the applied magnetic field. The inset shows a sketch of our sample. The shape of the overall loop reflects the wellseparated switching field distributions of the HL and SL, respectively. Two minor loops in the first and third quadrants in Fig. 1 (solid lines) resemble the reversal of the SL. The SL loops shown in Fig. 1 have been measured within a field range of $-100 \leq \mu_{0} H \leq 100 \mathrm{mT}$ when the HL magnetization is closely below its saturation. As noticed from the figure the position of the SL loops are shifted along the $m$ axis due to the remanent magnetic moment $m_{r}$ of the HL and along the field axis due to the $\mathrm{Ru}$ mediated interlayer exchange field $\mu_{0} H_{B} \propto m_{r}{ }^{15,17}$

The right frame of Fig. 1 shows several schematics that are depicting the evolution of the domain structures in the HL during typical experiment via arrows representing the local HL magnetization. These HL magnetization states correspond to the initialization [(a) and (b)] of the measurement process and subsequent SL training cycles [(c) first cycle and (d) after large number of cycles]. The initialization involves first saturation at $\mu_{0} H_{\text {sat }}=1 \mathrm{~T}$ such that the HL and SL magnetizations are completely aligned along the applied magnetic field. In a second step, a set field $-\mu_{0} H_{\text {sat }}<\mu_{0} H_{\text {set }}$ $<\mu_{0} H_{\mathrm{C} 1}$ is applied where $\mu_{0} H_{\mathrm{C} 1}$ is the negative coercive field of the overall loop (Fig. 1 shows an example for $\left.\mu_{0} H_{\text {set }}=-400 \mathrm{mT}\right)$. This set field partially demagnetizes the 


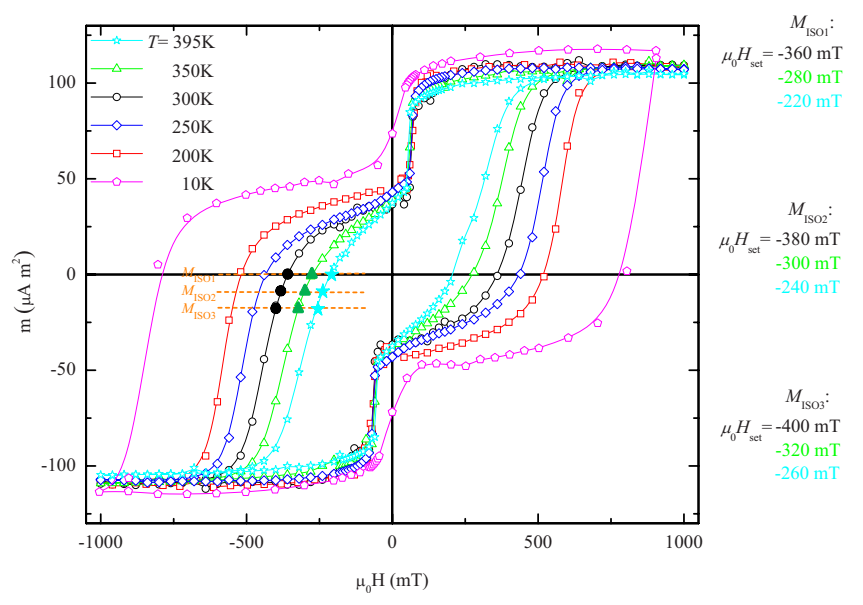

FIG. 2. (Color online) Overall magnetic hysteresis loops $m$ vs $\mu_{0} H$ at different temperatures $T=395,350,300,250,200$, and 10 $\mathrm{K}$. The three broken lines show the set fields producing isomagnetic HL domain states $M_{\mathrm{ISO} j}$ with $j=1,2$, and 3 .

HL and brings it in a domain state as shown in schematic (b). This partial HL demagnetization finalizes the initialization of the TE. Subsequently we measure the SL hysteresis loops in a magnetic-field range of $0<\mu_{0} H<100 \mathrm{mT}$ leaving the HL magnetization virtually unperturbed. Upon consecutive SL magnetization reversal, the HL interface spins are dragged back closer to the equilibrium spin configuration bringing the HL domain state closer to uniform HL magnetization. Therefore the HL quasiequilibrium which is reached in the limit of a large number of SL magnetization reversals has an increased magnetization with respect to the initial state of the training cycle. The schematics (c) and (d) resemble the HL domain states after first and a large number $n$ of SL hysteresis loops, respectively.

Figure 2 shows the overall magnetic hysteresis loops $m$ vs $\mu_{0} H$ at different temperatures $10 \leq T \leq 395 \mathrm{~K}$. All measurements are done with the help of a superconducting quantum interference device (SQUID) magnetometer (MPMS-XL, Quantum Design). Magnetic fields are applied within the easy anisotropy plane of the sample. As expected, the overall hysteresis loop broadens with decreasing temperature since domain-wall pinning is more effective when thermal activation is reduced. ${ }^{35}$ Note that the HL magnetization did not reach full saturation during the overall loop at $T=10 \mathrm{~K}$. As a consequence the overall loop shows a small asymmetry and, consistent with our training data, the SL magnetization reversal is broadened for a partially demagnetized HL.

In addition to the overall loops, Fig. 2 displays three horizontal lines which are isomagnetizations intercepting the loops at $M_{\mathrm{ISO} 1}=0, M_{\mathrm{ISO} 2}=-9.0$, and $M_{\mathrm{ISO} 3}=-18.0 \mathrm{nA} \mathrm{m}$. These isomagnetization lines define our specific experimental protocols of training initializations. We group those initializations at temperatures $T=300,350$, and $395 \mathrm{~K}$ together which belong to the same isomagnetization line. By doing so we obtain groups of data sets labeled by $j=1,2,3$. Different temperatures within a group refer to various HL states initialized according to one of the isomagnetization lines $M_{\mathrm{ISO} j}$. Figure 2 allows us to assign the set fields $\mu_{0} H_{\text {set }}=-360$, -280 , and $-220 \mathrm{mT}$ for group 1 which gives rise to $M_{\mathrm{ISO} 1}$
$=0$ at $T=300,350$, and $395 \mathrm{~K}$, respectively. Analogously $\mu_{0} H_{\text {set }}=-380,-300$, and $-240 \mathrm{mT}$ are the set fields for the initializations in group 2 and $\mu_{0} H_{\text {set }}=-400,-320$, and -260 mT correspond to group 3. Points $M_{\mathrm{ISO} j}\left(\mu_{0} H_{\text {set }}\right)$ are displayed as solid symbols for $j=1,2,3$. The grouping into isomagnetization initializations is necessary because our theoretical description requires the knowledge of the initial and the quasiequilibrium magnetization states of the HL as important inputs. In order to get data points which allow for comparison it is mandatory to start with an identical initial magnetization state of the HL.

Figure 3 shows the cycle-dependent evolution of the SL hysteresis loops reflecting typical training behavior of our all FM bilayer at four different temperatures in group $M_{\text {ISO3 }}$. The first ( $n=1$, squares), second ( $n=2$, circles), sixth $(n=6$, diamonds), and 15th ( $n=15$, triangles) hysteresis loops of the SL reveal a clear cycle-dependent relative shift along the field axis. The $n$ dependence is most pronounced for $T$ $=395 \mathrm{~K}$. It can be quantified by the relative change in the bias field $\Delta H_{B}^{\max } / H_{B}(n=1):=\left[H_{B}(n=15)-H_{B}(n=1)\right] / H_{B}(n$ =1) which is $2.0 \%$ at $T=395 \mathrm{~K}, 1.5 \%$ at $T=350 \mathrm{~K}, 0.6 \%$ at $T=300 \mathrm{~K}$, and experimentally not resolvable at $T=200 \mathrm{~K}$ for $M_{\mathrm{ISO} 3}$ initialization. $\Delta H_{B}^{\max } / H_{B}(n=1)$ is nonzero even below $T=300 \mathrm{~K}$ but is rapidly dropping with decreasing temperature due to reduced thermal assistance of the triggered relaxation dynamics.

Figure 4 shows the detailed analysis, $\mu_{0} H_{B}$ vs $n$, of the SL training loops at $T=300,350$, and $395 \mathrm{~K}$ for $M_{\mathrm{ISO} 3}$ initialization. The $n$ dependence of $\mu_{0} H_{B}$ reflects the tendency of the HL to approach its quasiequilibrium of increased magnetization on subsequently cycled SL loops. The circles are the experimental data and the lines are the least-square fits of Eq. (5). Its theoretical background will be discussed later in the text. It is observed that the change in $\mu_{0} H_{B}$ is more pronounced for lower $n$ and it attains saturation for higher $n$.

It is the aim of the presented work to evidence that we achieve consistent description of all our experimental data with our theory of the TE. Particular emphasis lies on the understanding of the temperature dependence of the rate of change in $\mu_{0} H_{B}$ vs $n$ which up to now entered the theory as a free-fitting parameter only. Our Landau-type theory provides a functional form of the latter.

\section{THEORY AND ANALYSIS OF EXPERIMENTAL RESULTS}

The TE originates from the nonequilibrium nature of the spin structure in the pinning layer ${ }^{23,25,36-39}$ reflecting the gradual recovery of equilibrium triggered by consecutive hysteresis loops of the SL. Significant TE is achieved only when a set field drives the HL far out of saturation into a domain state. Consecutively cycled loops of the SL then trigger partial relaxation of the HL back toward saturation. Recently this mechanism has been experimentally evidenced. ${ }^{17}$ In the framework of this physical picture, the TE in all FM bilayers has been described theoretically by means of the discretized Landau-Khalatnikov (LK) equation,

$$
\frac{S(n+1)-S(n)}{\tau}=-\frac{1}{\xi} \frac{\partial \Delta F}{\partial S} .
$$

Here $S$ is the interface magnetization of the HL, $\tau$ and $\xi$ are the time and damping constants, respectively, ${ }^{24}$ and $\Delta F$ is the 

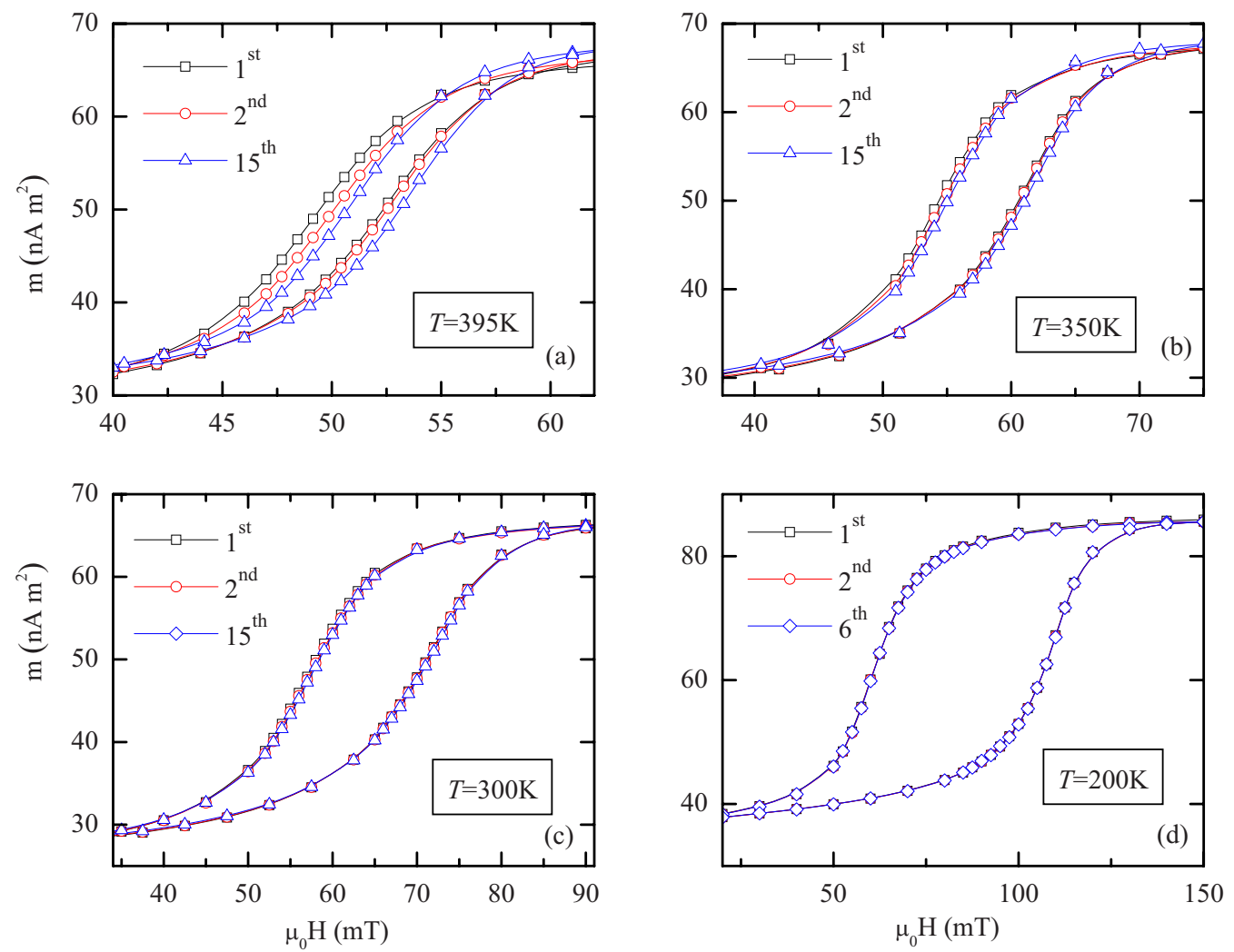

FIG. 3. (Color online) First (squares), second (circles), sixth (diamonds), and 15th (triangles) SL training hysteresis loops $m$ vs $\mu_{0} H$ at three different temperatures $T=395,350,300$, and $200 \mathrm{~K}$ for isomagnetization $M_{\mathrm{ISO} 3}$ set fields after saturating the bilayer sample at $\mu_{0} H_{\text {sat }}=1 \mathrm{~T}$.

nonequilibrium free energy of the HL. $\Delta F$ quantifies the free energy increase when the HL magnetization $M$ deviates from its quasiequilibrium value $M_{e}$. The magnetization $M$ which plays the role of the order parameter allows us to express the free energy in terms of Landau-type series expansions. The overall HL magnetizations, $M$ and $S$, are proportional since $\partial M / \partial z=0$ is a reasonable assumption for all positions $(x, y)$ in the sample plane. The derivative $-\partial \Delta F / \partial S$ can be interpreted as a force that drives the HL domain state back toward the quasiequilibrium state of magnetization $M_{e}$. Hence, Eq. (1) is a discretized form of the equation of motion for $S$ in the regime of overcritical damping. ${ }^{33}$ Since $\mu_{0} H_{B}=c_{1} S$ and $M=c_{2} S$ we express the free energy in terms of $M$ and use later $\mu_{0} H_{B}(n)=\frac{c_{1}}{c_{2}} M(n)$, with $c_{1,2}=$ const.

Note that the description of dynamics via the LandauKhalatnikov approach is unusual in magnetism but well established in ferroelectricity. ${ }^{40,41}$ Typically magnetization dynamics is described by the Landau-Lifshitz-Gilbert equation where an effective magnetic field creates a torque. ${ }^{42,43}$ This torque and a damping term together change the orientation of the magnetization vector. Here, however, the integral magnetization of the pinning layer is nonconserved since changes in the domain pattern are accompanied by changes in the overall magnetization. Relaxation of a nonconserved order parameter is dynamics of the model A type within the Hohenberg and Halperin ${ }^{44}$ classification schema and known to be described by the Landau-Khalatnikov equation. ${ }^{45}$ It has been explicitly shown for the simple case of a perfect ferro- magnet with a regular array of up and down domains that the connection between the dynamic behavior and the domain structure is consistent with our Landau-Khalatnikov approach leading to Eq. (1). ${ }^{46}$ Note that the LLG approach when embedded in a micromagnetic simulation which divides a sample into homogeneously magnetized interacting finite elements or grains that is by all means capable of describing domain effects and is able to fully explain nonuniform magnetization reversal and realistic hysteresis loops. Our aim here is, however, a simple analytic approach that catches the essentials and allows for intuitive interpretation. For this purpose our integral view on the overall magnetization of the pinning layer with the help of the LandauKhalatnikov approach is useful.

In our recent paper $^{17}$ we derived the functional form $\mu_{0} H_{B}=\mu_{0} H_{B}(n)$ from Eq. (1) using the Landau-type freeenergy expansion,

$$
F=F_{0}+\left.\frac{1}{2} \frac{\partial^{2} F}{\partial M^{2}}\right|_{M=M_{e}}\left(M-M_{e}\right)^{2},
$$

in the vicinity of the quasiequilibrium magnetization, $M_{e}$, attained by the HL after a large number of SL hysteresis loops. A straightforward result using Eqs. (1) and (2) and the proportionalities above is the implicit sequence,

$$
H_{B}(n+1)=(K+1) H_{B}(n)-K H_{B}^{e},
$$

where 

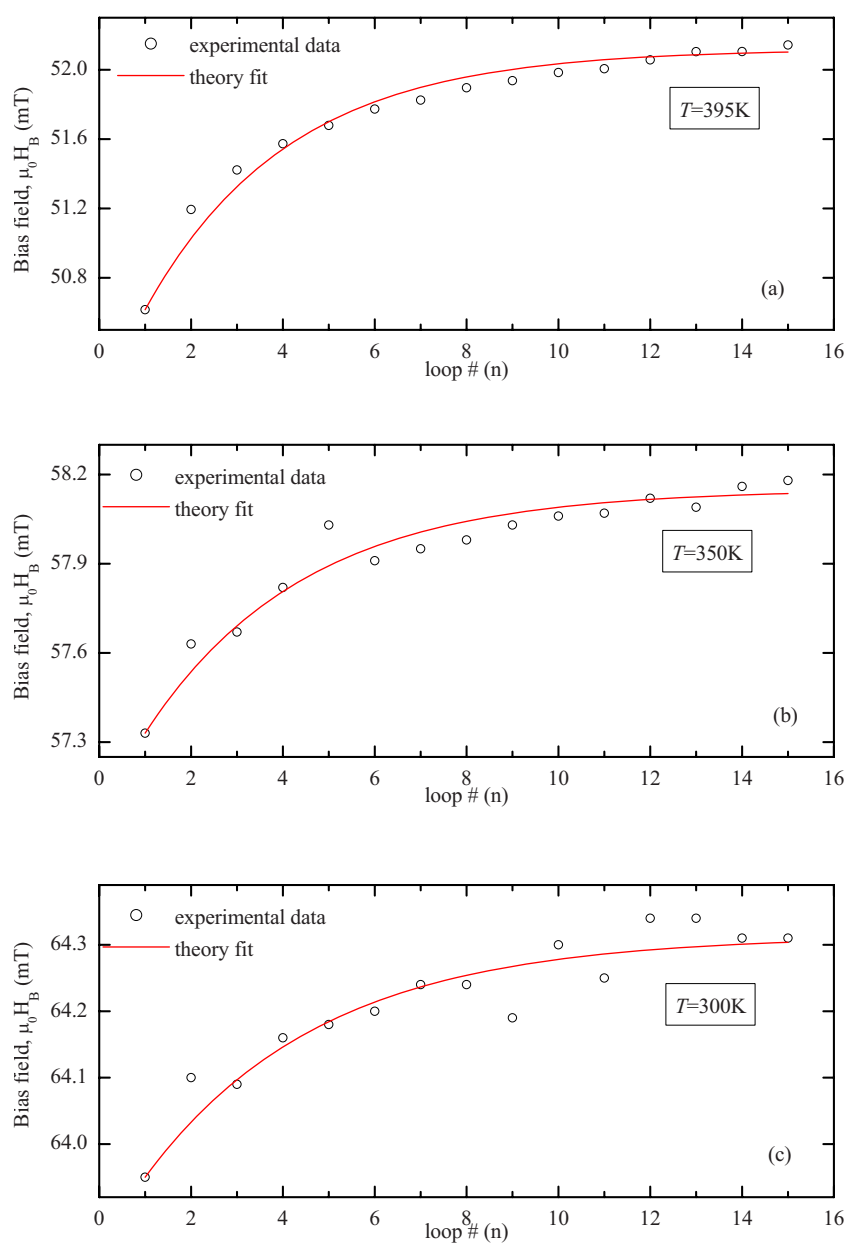

FIG. 4. (Color online) SL training effect $\mu_{0} H_{B}$ vs $n$ at $T=395$, 350 , and $300 \mathrm{~K}$ for initialization with isomagnetization $M_{\mathrm{ISO} 3}$ set fields after saturating the bilayer sample in $\mu_{0} H_{\text {sat }}=1 \mathrm{~T}$ at different temperatures. Circles are experimental data and lines represent least-squares fits of Eq. (5) to the data sets.

$$
K=-\left.\frac{\tau}{\xi} c_{2}^{2} \frac{\partial^{2} F}{\partial M^{2}}\right|_{M=M_{e}}<0 .
$$

Next we investigate the explict temperature dependence of $\left.\frac{\partial^{2} F}{\partial M^{2}}\right|_{M=M_{e}}>0$ and $\xi$.

The implicit sequence [Eq. (3)] can be transformed into the explicit fitting function, ${ }^{17}$

$$
\begin{aligned}
\mu_{0} H_{B}(n)= & (K+1)^{n-1}\left\{\mu_{0} H_{B}(n=1)\right. \\
& \left.-K \mu_{0} H_{B}^{e}\left[\frac{(K+1)^{n+1}-1}{K(K+1)^{n-1}}-(K+2)\right]\right\},
\end{aligned}
$$

appropriate to fit isothermal training data with $K$ as a crucial fitting parameter quantifying the rate of change in $\mu_{0} H_{B}(n)$. Equation (5) further involves the equilibrium bias field $\mu_{0} H_{B}^{e}=\mu_{0} H_{B}(n \rightarrow \infty)$ and the initial bias field $\mu_{0} H_{B}(n=1)$ obtained from the asymptotic behavior and the first point of the $\mu_{0} H_{B}$ vs $n$ data, respectively.

The objective of this paper is to extend our analysis qualitatively by deriving the explicit temperature dependence of $K$ which hitherto entered the theory as a fitting parameter only. We use Eq. (5) to obtain $K$ values for all of our training data $\mu_{0} H_{B}$ vs $n$ such as those shown exemplarily in Fig. 4. Leastsquares fits of the function $K(T)$ to these $K$ values will evidence the consistency of the theory. Subsequently we outline the derivation of the function $K(T)$ from Eq. (4).

In order to obtain the temperature dependence of $\left.\frac{\partial^{2} F}{\partial M^{2}}\right|_{M=M_{e}}$ which contains the temperature dependence of $K$ we compare Eq. (2) with the Landau expansion,

$$
F=F_{0}+\frac{1}{2} a M^{2}+\frac{1}{4} b M^{4}-H M,
$$

in the vicinity of $M=0$, where $a=a_{0}\left(T-T_{C}\right), T_{\mathrm{C}}$ is the Curie temperature of the HL and $a_{0}, b>0$ are the constants. From Eq. (6) we obtain,

$$
\left.\frac{\partial^{2} F}{\partial M^{2}}\right|_{M=M_{e}}=a+3 b M_{e}^{2}
$$

where $M_{e}$ is the solution of $a M_{e}+b M_{e}^{3}-H=0$ derived from $\left.\frac{\partial F}{\partial M}\right|_{M=M_{e}}=0$. Since the magnetic fields applied during the training cycles are small in comparison to the HL coercive fields the Zeeman term in Eq. (6) is negligible and the equilibrium magnetization $M_{e}$ can be expressed by the simple Landau expression $M_{e}=\sqrt{-a / b}$ allowing us to simplify expression (7) which then reads $\left.\frac{\partial^{2} F}{\partial M^{2}}\right|_{M=M_{e}}=2 b M_{e}^{2}=2 a_{0}\left(T_{c}-T\right)$. Substituting the latter expression into Eq. (4) we obtain,

$$
K=-\frac{\tau}{\xi} c_{2}^{2} a_{0}\left(T_{C}-T\right)
$$

Note that the decreasing accuracy of the simple Landau expression away from $T_{c}$ is compensated to a large extent by the strong temperature dependence of the damping constant, $\xi$, resulting in $K(T \rightarrow 0) \rightarrow 0$ independent of the specific functional form of $M_{e}(T)$. It can be shown that a mean-field solution for $M_{e}(T)$ yields very similar results for $K$ vs $T$ while the advantage of a simple analytic form of the results is lost, however.

The damping constant is known to be temperature dependent in other ferroic systems such as organic thin-film ferroelectrics ${ }^{45}$ having the functional form,

$$
\xi \propto \sqrt{T} \exp \left(\frac{2 U}{k T}\right),
$$

with $U$ being an energy barrier. The latter has the microscopic interpretation of a dipole/spin-flip energy. Using mean-field arguments this energy is given by $U=\left\langle z J s^{2}\right\rangle$, where $z$ is the number of nearest neighbors, $J$ is the exchange energy, $s$ is the spin quantum number, and $\langle\ldots\rangle$ denotes an average over the distribution of local configurations in the pinning layer alloy CoPtCrB. In mean-field approximation ${ }^{47}$ $U$ is related to $T_{C}$ via $U=3 s^{2} k_{B} T_{C} /[s(s+1)]$. In order to estimate an effective value of $s$ for the alloy $\mathrm{CoPtCrB}$ we recall the Slater-Pauling curve and in particular the strong deviations from the latter for Co-alloys involving elements which are two atomic numbers or more apart such as $\mathrm{Co}-\mathrm{Cr}$ for instance. ${ }^{48}$ Taking the strong suppression of the atomic magnetic moment in Co-alloys into account we use $s=1 / 2$ to 


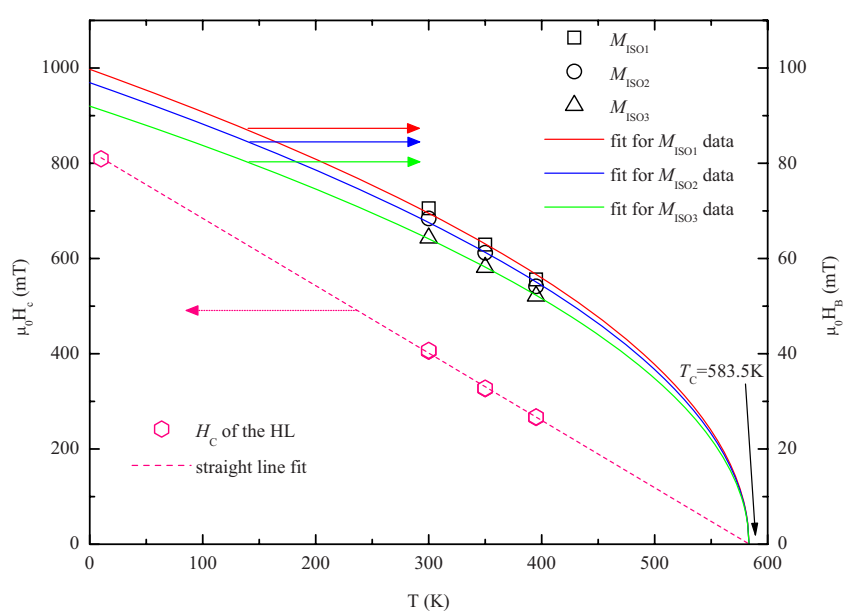

FIG. 5. (Color online) HL coercivity $\mu_{0} H_{C}$ vs $T$ (left axis). Hexagons represent experimental data, the dotted line is an empirical linear best fit. Its extrapolation to $\mu_{0} H_{C}=0$ provides an estimate of the HL Curie temperature $T_{C}=583.5 \mathrm{~K}$. The right axis shows the equilibrium bias field $\mu_{0} H_{B}^{e}$ vs $T$ for all three isomagnetization set fields. Squares $\left(M_{\mathrm{ISO} 1}\right)$, circles $\left(M_{\mathrm{ISO} 2}\right)$, and triangles $\left(M_{\mathrm{ISO} 3}\right)$ are the experimental data. Lines are single parameter best fits of Eq. (11).

obtain $U=k_{B} T_{C}$. Using this result for the energy barrier and substituting Eq. (9) into Eq. (8) we obtain

$$
K=-\frac{P}{\sqrt{T}} e^{-2 T_{C} / T}\left(T_{C}-T\right),
$$

where $P>0$ is a free parameter.

The Curie temperature, $T_{C}$, of the HL enters Eq. (10) and, therefore, makes it preferable to have independent experimental access to its value. We estimate $T_{C}$ experimentally from the temperature dependence of the HL coercivity $\mu_{0} H_{C}$ vs $T$ through extrapolation of the data to the intercept with the temperature axis. The left axis of Fig. 5 shows the coercivity data $\mu_{0} H_{C}$ vs $T$ of the HL. The latter are obtained from the overall hysteresis loops displayed in Fig. 2. Note, however, that the apparent HL coercivity, $H_{C}^{\text {broad }}$, has contributions from the intrinsic $\mathrm{HL}$ coercivity, $H_{C}$, and from a coupling-induced HL loop broadening. $H_{C}^{\text {broad }}$ itself is obtained from the overall loops after subtracting the SL magnetization. Correcting with respect to the coupling-induced broadening is a small but somewhat involved effect. The SL/HL coupling at $H_{C}$ of the $\mathrm{HL}$ is given by the bias field created by the fully saturated SL. Thus the bias coming from the SL and affecting the HL coercivity has to be related to the bias onto the SL that a fully magnetized HL generates $H_{B}^{\max }$. Quantitatively the effect on the HL depends on the ratio of the SL/HL magnetizations and hence on the weighting factor $m_{\mathrm{SL}} / m_{\mathrm{HL}}$. The SL coupling contribution has to be subtracted to get the genuine HL coercivity. This correction is done by using $H_{C}=H_{C}^{\text {broad }}-\left[H_{B}^{\mathrm{max}} m_{\mathrm{SL}} / m_{\mathrm{HL}}\right]$. The hexagons in Fig. 5 are experimental $\mu_{0} H_{C}$ vs $T$ data. The corresponding dotted line is the best linear fit. Extrapolation down to $\mu_{0} H_{C}=0$ yields the HL Curie temperature $T_{C}=583.5 \mathrm{~K}$. The linear extrapolation is the best we can do in the absence of a rigorous theory for $\mu_{0} H_{C}$ vs $T$. In fact the simple Landau

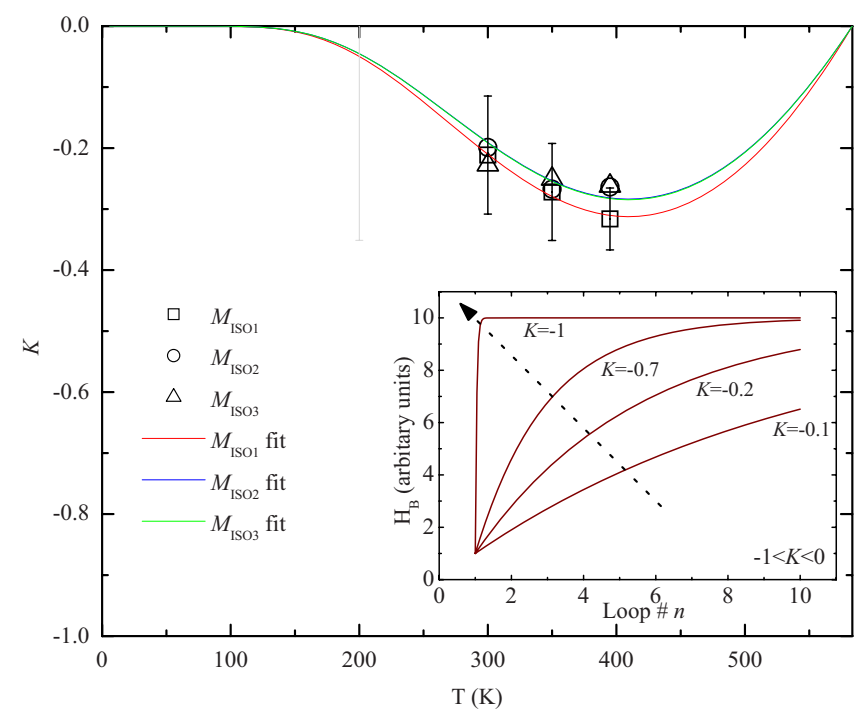

FIG. 6. (Color online) $K$ vs $T$ for the three isomagnetization set fields. Initializations $M_{\mathrm{ISO} 1}$ (squares), $M_{\mathrm{ISO} 2}$ (circles), and $M_{\mathrm{ISO} 3}$ (triangles) are the experimental data. Lines are single parameter best fits of Eq. (10). Representative error bars are shown for $M_{\mathrm{ISO} 1}$ and calculated from Eq. (13). The inset shows simulated plots of Eq. (5) visualizing the role of $K$ in the characteristics of $H_{B}$ vs $n$. Value of $K$ decreases from close to zero toward $K=-1$ in the direction of the arrow. Identical arbitrary values of the first and the equilibrium bias value are used for all simulated curves.

expression $a M_{e}+b M_{e}^{3}-H=0$ predicts the nonlinear behavior $H_{C}=\sqrt{\frac{-4\left[a_{0}\left(T-T_{C}\right)\right]^{3}}{27 b}}$ which approaches the $T$ axis slower than the linear extrapolation implying a higher value of $T_{C}$. However, the intrinsic coercivity considered in the latter expression is never relevant in real ferromagnets. In addition $T_{C}$ $=583.5 \mathrm{~K}$ obtained from the linear extrapolation is strongly supported by the fits of $\mu_{0} H_{B}^{e}$ vs $T$ discussed in the next paragraph.

The right axis of Fig. 5 shows the equilibrium bias fields $\mu_{0} H_{B}^{e}$ vs $T$ for the initializations $M_{\mathrm{ISO} 1}$ (squares), $M_{\mathrm{ISO} 2}$ (circles), and $M_{\mathrm{ISO} 3}$ (triangles). The lines represent single parameter fits of the function

$$
\mu_{0} H_{B}^{e}(T)=\mu_{0} H_{B}^{e}(T=0) \sqrt{\frac{T_{C}-T}{T_{C}}},
$$

yielding $\quad \mu_{0} H_{B}^{e}(T=0)=99.73 \pm 0.97, \quad 96.93 \pm 0.82, \quad$ and $92.01 \pm 0.17 \mathrm{mT}$ for $M_{\mathrm{ISO} 1}, M_{\mathrm{ISO} 2}$, and $M_{\mathrm{ISO} 3}$, respectively. Note that the successful fit of Eq. (11) reconfirms the applicability of the simple Landau expression for the temperature dependence of the HL magnetization which leads to Eq. (8).

Finally Fig. 6 shows all $K$ vs $T$ data obtained from leastsquare fits of Eq. (5) to the experimental $\mu_{0} H_{B}$ vs $n$ data (see lines for fits and circles for typical training data in Fig. 4). The experimental $K$ data in Fig. 6 originate from training initializations $M_{\mathrm{ISO} 1}$ (squares), $M_{\mathrm{ISO} 2}$ (circles), and $M_{\mathrm{ISO} 3}$ (triangles). Lines represent the results of a best fits of Eq. (10) to the respective data set where $P$ is the single free fitting with $P=0.626 \pm 0.009, \quad 0.570 \pm 0.023$, and $0.572 \pm 0.0396 \mathrm{~K}^{-1 / 2}$ for $M_{\mathrm{ISO} 1}, M_{\mathrm{ISO} 2}$, and $M_{\mathrm{ISO} 3}$, respectively. As a typical example we show error bars for the $M_{\text {ISO1 }}$ 
data. Next we briefly describe how those error bars are obtained.

While the $K$ values shown in Fig. 6 are determined from best fits of Eq. (5) to respective training data, an alternative determination of optimized $K$ values is obtained from the expression

$$
K=\frac{\sum_{n=1}^{N-1}\left[H_{B}(n)-H_{B}^{e}\right]\left[H_{B}(n+1)-H_{B}(n)\right]}{\sum_{n=1}^{N-1}\left[H_{B}(n)-H_{B}^{e}\right]^{2}} .
$$

Here the $H_{B}^{e}$ is an input obtained from the fit of Eq. (5). Equation (12) is derived from a least-squares condition using Eq. (3). Expression (12) is used to calculate the standard deviation $S_{K}$ of $K$ from Gauss' law of error propagation which reads as

$$
S_{K}=\sqrt{\sum_{n=2}^{N-1}\left(\frac{\partial K}{\partial H_{B}(n)} \Delta H_{B}(n)\right)^{2}},
$$

where $\Delta H_{B}(n)$ is the error in the bias field of the $n$th training loop. The derivatives entering $S_{K}$ are calculated from Eq. (12) and read as

$$
\begin{aligned}
\frac{\partial K}{\partial H_{B}(n)}= & \frac{\left[H_{B}(n-1)+H_{B}(n+1)-2 H_{B}(n)\right]}{\sum_{n=1}^{N-1}\left[H_{B}(n)-H_{B}^{e}\right]^{2}} \\
& -2 K \frac{\left[H_{B}(n)-H_{B}^{e}\right]}{\sum_{n=1}^{N-1}\left[H_{B}(n)-H_{B}^{e}\right]^{2}}
\end{aligned}
$$

With $\Delta \mu_{0} H_{B}(n) \approx 0.1 \mathrm{mT} \forall n$ it is straight forward to numerically determine $S_{K}$. The results of this analysis are shown for one example $\left(M_{\mathrm{ISO} 1}\right)$ in Fig. 6 as error bars. Note that the magnitude of the error bars increases with decreasing temperature. When applying the same analysis to the $T$ $=200 \mathrm{~K}$ data set where $\mu_{0}\left[H_{B}^{e}-H_{B}(1)\right] \approx 0.1 \mathrm{mT}$ is extremely small $S_{K}=0.3$ in turn becomes even significantly larger than the theoretically expected value of $|K|=0.05$. Note that this increase in the error bar takes place despite the fact that the absolute accuracy of the bias fields remains $\Delta \mu_{0} H_{B}(n) \approx 0.1 \mathrm{mT}$ [see Fig. 3(d)]. Hence it is obvious that any attempt to determine $K$ values at low temperatures where $\Delta H_{B}=H_{B}^{e}-H_{B}(1) \rightarrow 0$ will become experimentally virtually impossible.

The inset of Fig. 6 provides an intuitive understanding of the role of $K$ for the characteristics of the TE. A family of curves is displayed where $K$ is varied within the range of $-1 \leq K \leq 0$. This interval defines the range of convergence for the geometrical series involved in the transformation of the implicit sequence (3) into the explicit Eq. (5). The value of $K$ changes from 0 to -1 along the direction of displayed arrow. Inspection of Eq. (3) shows that $K=0$ yields $H_{B}(n$ $+1)=H_{B}(n)$ which means no training at all. Note that this does not imply that the bias field has to be zero. Similarly $\Delta H_{B}=H_{B}^{e}-H_{B}(1) \rightarrow 0$ does not necessarily imply $K \rightarrow 0$. $K$
$=-1$ in turn yields $H_{B}(n+1)=H_{B}^{e} \forall n \geq 1$ which means a steplike change in the bias field between the first two points and zero training for $n>2$. Intuitively $K\left(T \geq T_{C}\right)=0$ has to be fulfilled because $H_{B}(n+1)=H_{B}^{e}=0 \forall n \geq 1$ at $T \geq T_{C}$ reflecting the absence of biasing and, hence, training. Similarly $K(T=0)=0$ holds. Here, however, $K(T=0)=0$ reflects the nontrivial situation where a nonzero-bias field can be accompanied by zero TE. Instead of zero-bias field associated with zero pinning layer magnetization a nonzero pinning layer magnetization can be frozen in at $T=0$. Domain walls are pinned and the absence of thermal activation keeps the pinning layer in the initial domain state. In the framework of Eq. (3) this freezing behavior is reflected by a diverging damping constant [see Eq. (9)] which give rise to $K=0$. In addition the $K=0$ state at $T=0$ is approached with $d K /\left.d T\right|_{T=0}=0$ similarly to the asymptotic behavior of equilibrium thermodynamic properties obeying the third law of thermodynamics.

It is hard to imagine any arbitrary single parameter fitting function which is consistent with the constraints $K(T=0)$ $=0, d K /\left.d T\right|_{T=0}=0$, and $K\left(T=T_{C}\right)=0$ providing the quality of the fits as shown in Fig. 6. Moreover, the fitting parameters of Eqs. (10) and (11) reflect the ratio $P_{\mathrm{ISO} 1} / P_{\mathrm{ISO} 2}=1.10$ $\approx\left[H_{B}^{e}(T=0, \mathrm{ISO} 1) / H_{B}^{e}(T=0, \mathrm{ISO} 2)\right]^{2}=1.06$ as expected from Eqs. (4) and (10) and the proportionality between $H_{B}^{e}$ and $M_{e}$.

\section{CONCLUSIONS}

We have shown that bilayers of antiferromagnetically coupled hard and soft ferromagnetic thin films have prototypical properties providing fundamental understanding of exchange bias and its training effect. We demonstrated that in far reaching analogy to antiferromagnetic/ferromagnetic exchange bias heterolayers quantitative understanding of the temperature dependence of the training effect is achieved. Large training effects reflected by the parameter $-1<K<0$ require thermal activation allowing for triggered changes in the domain structure of the pinning layer but at the same time sufficient thermal stability of the pinning layer magnetization. This competition between thermal activation and stability creates maximum training effects at $T=T_{C}(\sqrt{41}$ $-5) / 2$. The successful modeling of the temperature dependence of the training effect in our all FM bilayer system confirms the consistent description of training behavior in the discretized Landau-Khalatnikov approach.

\section{ACKNOWLEDGMENTS}

Work at UNL was supported by NSF through Grant No. DMR-0547887, by the Nebraska Research Initiative (NRI), and by the MRSEC Program of the NSF through Grant No. DMR-0213808. Work at nanoGUNE acknowledges funding from the Department of Industry, Trade, and Tourism of the Basque Government and the Provincial Council of Gipuzkoa under the ETORTEK program, Project No. IE06-172, as well as from the Spanish Ministry of Science and Education under the Consolider-Ingenio 2010 program, Project No. CSD2006-53. 
*cbinek2@unl.edu

${ }^{1}$ W. H. Meiklejohn and C. P. Bean, Phys. Rev. 102, 1413 (1956).

${ }^{2}$ W. H. Meiklejohn and C. P. Bean, Phys. Rev. 105, 904 (1957).

${ }^{3}$ J. Nogués and I. K. Schuller, J. Magn. Magn. Mater. 192, 203 (1999).

${ }^{4}$ A. Berkowitz and K. Takano, J. Magn. Magn. Mater. 200, 552 (1999).

${ }^{5}$ T. J. Moran, J. M. Gallego, and I. K. Shuller, J. Appl. Phys. 78, 1887 (1995).

${ }^{6}$ J. Nogués, D. Lederman, T. J. Moran, I. K. Shuller, and K. V. Rao, Appl. Phys. Lett. 68, 3186 (1996).

${ }^{7}$ J. Ventura, J. P. Araujo, J. B. Sousa, A. Veloso, and P. P. Freitas, Phys. Rev. B 77, 184404 (2008).

${ }^{8}$ I. S. Jiang, E. E. Fullerton, C. H. Sowers, A. Inomata, S. D. Bader, A. J. Shapiro, R. D. Shull, V. S. Gornakov, and V. I. Nikitenko, IEEE Trans. Magn. 35, 3229 (1999).

${ }^{9}$ M. Sorescu, A. Grabias, and M. Valeanu, IEEE Trans. Magn. 39, 2959 (2003).

${ }^{10}$ E. E. Fullerton, J. S. Jiang, M. Grimsditch, C. H. Sowers, and S. D. Bader, Phys. Rev. B 58, 12193 (1998).

${ }^{11}$ J. E. Shield, J. Zhou, S. Aich, V. K. Ravindran, Ralph Skomski, and D. J. Sellmyer, J. Appl. Phys. 99, 08 B508 (2006).

${ }^{12}$ M. Kiwi, J. Magn. Magn. Mater. 234, 584 (2001).

${ }^{13}$ J. I. Hong, T. Leo, D. J. Smith, and A. E. Berkowitz, Phys. Rev. Lett. 96, 117204 (2006).

${ }^{14}$ Ch. Binek, X. Chen, A. Hochstrat, and W. Kleemann, J. Magn. Magn. Mater. 240, 257 (2002).

${ }^{15}$ A. Berger, D. T. Margulies, and H. Do, Appl. Phys. Lett. 85, 1571 (2004).

${ }^{16}$ A. Berger, Ch. Binek, D. T. Margulies, A. Moser, and E. E. Fullerton, Physica B (Amsterdam) 372, 168 (2006).

${ }^{17}$ Ch. Binek, S. Polisetty, X. He, and A. Berger, Phys. Rev. Lett. 96, 067201 (2006).

${ }^{18} \mathrm{~S}$. Sahoo, T. Mukherjee, K. D. Belashchenko, and Ch. Binek, Appl. Phys. Lett. 91, 172506 (2007).

${ }^{19}$ J. Nogués, J. Sort, S. Suriñach, J. S. Muñoz, M. D. Baró, J. F. Bobo, U. Lüders, E. Haanappel, M. R. Fitzsimmons, A. Hoffmann, and J. W. Cai, Appl. Phys. Lett. 82, 3044 (2003).

${ }^{20}$ D. Paccard, C. Schlenker, O. Massenet, R. Montmory, and A. Yelon, Phys. Status Solidi 16, 301 (1966).

${ }^{21}$ C. Schlenker, S. S. P. Parkin, J. C. Scott, and K. Howard, J. Magn. Magn. Mater. 54-57, 801 (1986).

${ }^{22}$ K. Zhang, T. Zhao, and M. Fujiwara, J. Appl. Phys. 89, 6910 (2001).

${ }^{23}$ H. Xi, R. M. White, S. Mao, Z. Gao, Z. Yang, and E. Murdock, Phys. Rev. B 64, 184416 (2001).

${ }^{24}$ S. Sahoo, S. Polisetty, Ch. Binek, and A. Berger, J. Appl. Phys. 101, 053902 (2007).
${ }^{25}$ A. Hoffmann, Phys. Rev. Lett. 93, 097203 (2004).

${ }^{26}$ S. Polisetty, S. Sahoo, and Ch. Binek, Phys. Rev. B 76, 184423 (2007).

${ }^{27}$ T. Hauet, J. A. Borchers, Ph. Mangin, Y. Henry, and S. Mangin, Phys. Rev. Lett. 96, 067207 (2006).

${ }^{28}$ M. Ali, C. H. Marrows, and B. J. Hickey, Phys. Rev. B 67, 172405 (2003).

${ }^{29}$ J. Keller, P. Miltényi, B. Beschoten, G. Güntherodt, U. Nowak, and K. D. Usadel, Phys. Rev. B 66, 014431 (2002).

${ }^{30}$ Ch. Binek, X. He, and S. Polisetty, Phys. Rev. B 72, 054408 (2005).

${ }^{31}$ H. Ohldag, A. Scholl, F. Nolting, E. Arenholz, S. Maat, A. T. Young, M. Carey, and J. Stöhr, Phys. Rev. Lett. 91, 017203 (2003).

${ }^{32}$ P. Kappenberger, S. Martin, Y. Pellmont, H. J. Hug, J. B. Kortright, O. Hellwig, and E. E. Fullerton, Phys. Rev. Lett. 91, 267202 (2003).

${ }^{33}$ Ch. Binek, Phys. Rev. B 70, 014421 (2004).

${ }^{34}$ A. Berger, O. Hovorka, G. Friedman, and E. E. Fullerton, Phys. Rev. B (to be published).

${ }^{35}$ Xi Chen, O. Sichelschmidt, W. Kleemann, O. Petracic, Ch. Binek, J. B. Sousa, S. Cardoso, and P. P. Freitas, Phys. Rev. Lett. 89, 137203 (2002).

${ }^{36}$ U. Nowak, K. D. Usadel, J. Keller, P. Miltényi, B. Beschoten, and G. Güntherodt, Phys. Rev. B 66, 014430 (2002).

${ }^{37}$ D. Suess, M. Kirschner, T. Schrefl, J. Fidler, R. L. Stamps, and J. V. Kim, Phys. Rev. B 67, 054419 (2003).

${ }^{38}$ M. D. Stiles and R. D. McMichael, Phys. Rev. B 60, 12950 (1999).

${ }^{39}$ S. Brems, D. Buntinx, K. Temst, C. Van Haesendonck, F. Radu, and H. Zabel, Phys. Rev. Lett. 95, 157202 (2005).

${ }^{40} \mathrm{~V}$. Stephanovich (private communication).

${ }^{41}$ L. Blinov, A. Bune, P. Dowben, S. Ducharme, V. Fridkin, S. Palto, K. Verkhovskaya, G. Vizdrik, and S. Yudin, Phase Transitions 77, 161 (2004).

${ }^{42}$ S. A. Michalski, J. Zhou, R. Skomski, and R. D. Kirby, J. Appl. Phys. 101, 09D115 (2007).

${ }^{43}$ E. G. Galkina, B. A. Ivanov, and V. A. Stephanovich, J. Magn. Magn. Mater. 118, 373 (1993).

${ }^{44}$ P. C. Hohenberg and B. I. Halperin, Rev. Mod. Phys. 49, 435 (1977).

${ }^{45}$ A. R. Massih and L. O. Jernkvist, J. Phys. Chem. Solids 65, 1193 (2004).

${ }^{46}$ J. Rajchenbach, J. Phys. C 21, L447 (1988).

${ }^{47}$ H. E. Stanley, Introduction to Phase Transition and Critical Phenomena (Oxford University Press, New York, 1971).

${ }^{48}$ R. M. Bozorth, Phys. Rev. 79, 887 (1950). 